\title{
Circuit training for 12 and under tennis players: an- court exercise proposal
}

\author{
Carlos Valle García
}

\section{ABSTRACT}

The transition from global exercises to more specific ones, using specific equipment and stimuli, is key for the physical conditioning of young tennis players. This article describes a proposal for oncourt agility, coordination, speed, injury prevention, power and balance exercises for developing 12year-old tennis players.
Key words: Circuit training, young players, physical conditioning.

Received: 06 February 2020

Accepted: 12 March 2020

Corresponding author: Carlos Valle García, Spain. Email: chals.vall@gmail.com

\section{INTRODUCTION}

Physical conditioning adapted to the needs of young tennis players is key, not only because of the importance of physical conditioning in modern tennis, but because of the need for multidisciplinary training in the physical qualities that tennis demands. Circuit training is a very efficient methodology for the physical conditioning of 12-year-old tennis players, since it is perfectly adaptable to the daily conditions that trainers and coaches manage at tennis clubs and academies. For examples of this kind of training with tennis players of different ages, see the work done by Dent (1996), Chu (1998), González (2003), Marques (2005), Mohanta, Kalra, \& Pawaria (2019) and Genevois (2019).

\section{BENEFITS AND CONSIDERATIONS}

Circuit training comprising of simultaneous workstations allows for specific control of the work and recovery times. Thus, the coach can manage the density of the load by means of these variables. When there are more players than stations, the work time of the first group doubles up as recovery time for the second group and vice versa. This kind of training helps create a high intensity working environment where the participation of players is active and constant, letting the coach control the equipment and structure the exercises in a clear and orderly fashion. At the beginning of the session, the coach must clearly explain and demonstrate the technique for the execution of the movements, the working and rest times, the sequence of the stations, and the maximum number of possible repetitions in an exercise (González, 2003).

Among other things, during this stage, the player must learn how to develop versatility, gain experience, improve the level of motor and mental skills, and improve the main specific capabilities for tennis. It is important to highlight that it is at the age of 12 when the two most important motor capabilities for tennis, agility and speed, can best be developed (Unierzyski, 2003).

\section{PRACTICAL APPLICATION}

Strength and physical conditioning for young tennis players implies progressive implementation of appropriate exercises for general physical development as well as more important and specific areas for tennis players. These exercises include jumping, throwing, receiving, strength, power, speed, coordination, balance, endurance, and flexibility (Kovacs, 2016).

This article presents an example of on- court circuit training for 12-year-old players, with 6 stations and 2 players working simultaneously per station. Physical qualities, like explosive strength (plyometric work), specific coordination to hit from an open stance, core strengthening (preventive work), balance, hand-eye coordination, agility, and running technique together with speed are worked. Working time: 15 seconds, the same as resting time, with 2 consecutive series (i.e. two lots of work and rest - $1 \mathrm{~min}$.) in each station, reaching a 1:1 work to rest ratio. The work and rest time will vary depending on the physical qualities of the players, their targets and their technical adaptation to the exercises. 


\section{Station 1: Plyometric work of lower limbs}

It has been demonstrated that the application of plyometric work to habitual tennis training seems to be a more appropriate stimulus for the improvement of physical qualities in young players when compared to training without plyometric practice. Thus, specific power training is suitable for increasing tennis explosive actions (Fernández-Fernández et al., 2016).

Equipment: $2 x$ high fences $(30-40 \mathrm{~cm})$ and $2 x$ low fences (10$20 \mathrm{~cm}$ ). The height of the fences may be modified depending on the physical characteristics of the players.

Protocol: The player jumps over the higher fences coordinating the impulse with the movement of the arms ( $A B K$ jump), followed by two jumps over the lower fences, with stretched knees and quick flex-extensions of the ankle joint at the maximum possible speed. The process is repeated until the end of the set. The coach must explain execution and landing techniques.

Direct connection to tennis: Power and changes of direction.

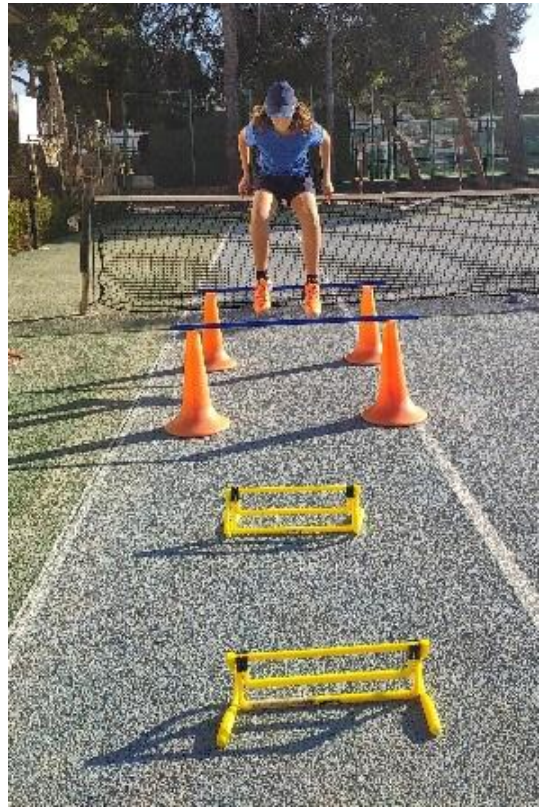

Figure 1. Station 1: plyometric work of lower limbs

Station 2: Receiving and throwing the Swiss ball in an open stance

Receiving and throwing skills are key for the learning and development of the new movement patterns. The combination of these skills along with adequate positioning for stroke execution can be properly trained with Swiss balls (Genevois, Reid \& Crespo, 2016).

Equipment: Swiss ball, 2 cones per player.

Protocol: In a $2 \mathrm{~m}$ per side square, two players $(A \& B)$ stand in opposite corners, player A will throw a Swiss ball in an arc- shaped flight path to the opposite cone at the signal: "NOW!" Player, B makes a cross first step, coordinating the lateral movement to catch the ball in an open stance, taking the ball at waist height, rotating the shoulders over the cone, simulating a back-swing position. Player $B$ always throws the Swiss ball towards the diagonal cone for the player to repeat the same reception and preparation process. After two shots each, player $A$ and player $B$ interchange the direction of their shots in order to catch on the other side.

Direct connection to tennis: Space-time coordination in stroke preparation.

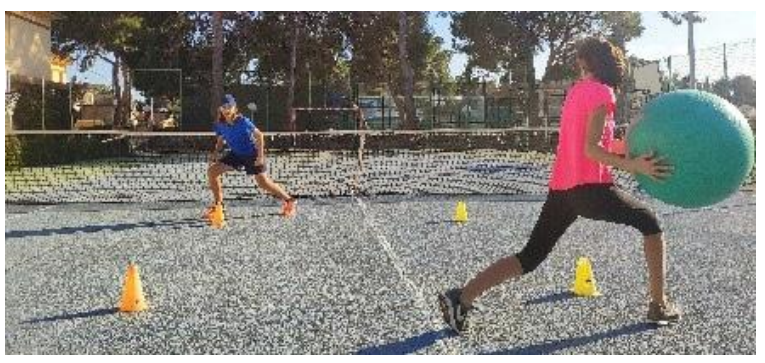

Figure 2. Station 2: receiving and throwing the Swiss ball in an open stance

Station 3: Reactive hand-eye coordination

Hand-eye coordination is a key quality for tennis. Here, the reaction must be trained by means of varied exercises (Dent, 1996).

Equipment: 1 tennis ball and 2 cones per player.

Protocol: The players (one holding a tennis ball) stand in a line with 1 cone at $1.5 \mathrm{~m}$. from them on each side perpendicular to the line. When the partner points at one of the two cones, the player must throw the tennis ball underarm to the partner but so that it bounces, touch the specific colour cone with their hands, and return to the centre before the ball has bounced for the second time.

Direct connection to tennis: Ocular-manual reaction with the bounce of the tennis ball.

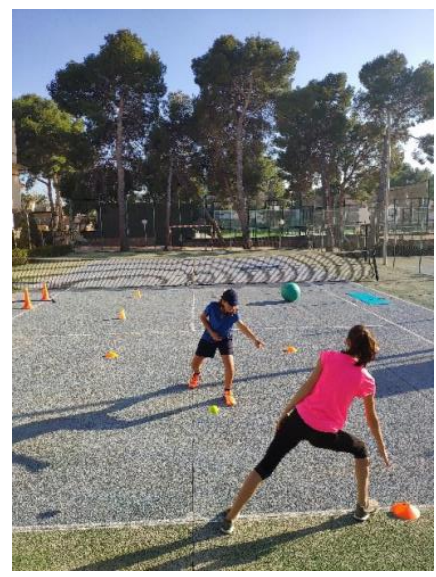

Figure 3. Station 3: Reactive hand-eye coordination 


\section{Station 4: Forward movement \& sprint technique}

Learning an appropriate movement technique is key for the efficient execution of strokes. Coordination between running and sprinting must be trained in development stages, but mainly, before the players are 12 years old, so that they acquire the correct foundations (Chu, 1998).

Equipment: 1 elastic rubber band and 3 cones.

Protocol: The player doing the exercise, with a band round their waist and facing away from their partner, tries to run away from their partner whilst the partner holds the ends of the band. The player lifts their knees 6 times, alternating, and at maximum speed, coordinating with the natural movements of the arms, trying to reach the furthest cone (cone at $1-2 \mathrm{~m}$, cone at $2-3 \mathrm{~m}$ ). After 2 sets, the player gets rid of the rubber band and makes a $5 \mathrm{~m}$ sprint with the running technique just employed in place.

Direct connection to tennis: Coordination in running and sprinting.

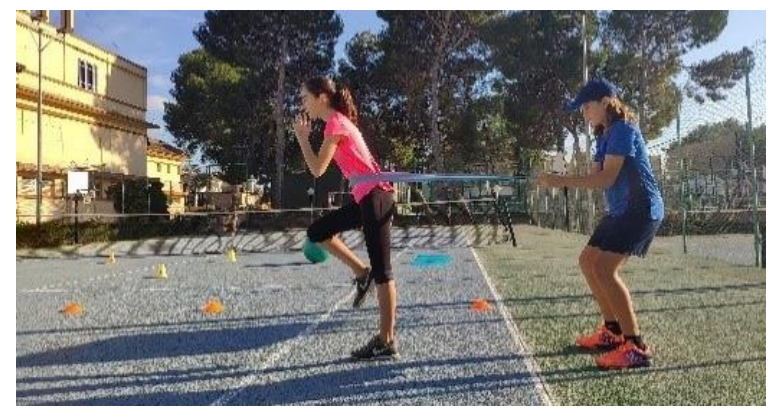

Figure 4. Station 4: Forward movement \& sprint technique

Station 5: Lateral plank with elastic rubber band with leg extension

Static balance is an indispensable motor skill for tennis, facilitated by muscular synergies that keep the player in an upright position and correct shape when the player is not in movement (Samson, Sandrey \& Hetrick, 2007).

Equipment: Mat, elastic rubber band - low tension.

Protocol: Player A takes a side plank position holding one end of the rubber band with the hand of the elevated arm while player B works on static balance on one leg. The rubber band is around the standing foot of the player balancing on one-leg. The player makes a complete set in plank position / balancing, changing sides / legs in the second set.

Direct connection to tennis: CORE, knee and ankle stability.
Station 6: Proactive dynamic balance with Swiss ball and unstable bases

Dynamic balance is the motor capability to keep the body in balance when the tennis player is in movement. The characteristics of tennis make this a key aspect for performance (Reid \& Schneiker, 2008).

Equipment: 6 rings, 1 Swiss ball and 2 Bosu Balance Trainers.

Protocol: Moving from platform/ring to another every few seconds, the player must keep balance inside each ring or unstable platform for 3 seconds on one leg, following the circuit and alternating tennis specific open and closed positions.

Direct connection to tennis: Body control and balance during strokes.

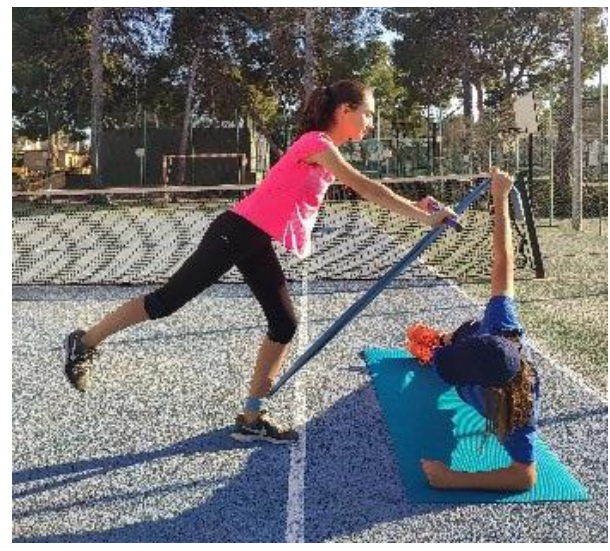

Figure 5. Station 5: Lateral plank with elastic rubber band with legextension

\section{CONCLUSIONS}

Circuit training is a highly recommended method to work different physical qualities simultaneously with a group or tennis players. It is possible to control the content, volume and intensity of the appropriate work in an easy and effective way. It may be directed by a single coach using basic material on the tennis court. Finally, its use improves the physical qualities in 12 and under players.

\section{REFERENCES}

Fernández-Fernández, J.; Sáez De Villarreal, E.; Sanz-Rivas, D.; \& Moya, M. (2016) The Effects of 8-Week Plyometric Training on Physical Performance in Young Tennis Players. Pediatric Exercise Science, 28, 77 -86, https://doi.org/10.1123/pes.2015-0019

Genevois, C. (2019). The importance of aerobic fitness for tennis: training and testing (part 2) ITF Coaching and Sport Science Review 79 (27): 16-18. 
Genevois, C., Reid, M., \& Crespo, M. (2016). The forehand shot in tennis: performance factors, functional analysis and practical implications, ITF Ltd., London.

Chu, D. A. (1998). On-Court circuit training for improving change of direction speed in tennis. ITF Coaching and Sport Science Review. Apr; 14-15.

Dent, P. (1996). Coordinate to accelerate. ITF Coaching and Sport Science Review, 8, 6-7.

Gonzalez, R. (2003). Circuit training for tennis, ITF Coaching \& Sport Science Review 31, 13-14.

Kovacs, M. S. (2016). Strength and Conditioning for the Young Tennis Player. In The Young Tennis Player (pp. 55-86). Springer, Cham, https://doi.org/10.1007/978-3-31927559-8 4

Marques, M. A. C. (2005). Strength training in adult elite tennis players, Strength and Conditioning Journal, 27(5), 34-38.

Mohanta, N., Kalra, S., \& Pawaria, S. (2019). A Comparative Study of Circuit Training and Plyometric Training on Strength, Speed and Agility in State Level Lawn Tennis Players. Journal of Clinical \& Diagnostic Research, 13(12), 5-10, https://doi.org/10.7860/JCDR/2019/42431.13348

Reid, M., \& Schneiker, K. (2008). Strength and conditioning in tennis: current research and practice. Journal of Science and Medicine in Sport, 11(3), 248-256, https://doi.org/10.1016/i.jsams.2007.05.002

Samson, K. M., Sandrey, M. A., \& Hetrick, A. (2007). A core stabilization training program for tennis athletes. Athletic Therapy Today, 12(3), 41-44.

Unierzyski, P. (2003). Planning and periodization for the 1214 years old tennis players. ITF Coaching \& Sport Science Review 31, 6-8, https://doi.org/10.1123/att.12.3.41

RECOMMENDED ITF TENNIS ACADEMY CONTENT (CLICK BELOW)

\section{ITF Academy}

Copyright (c) 2020 Carlos Valle García

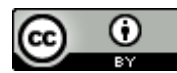

This text is under a Creative Commons BY 4.0 license

You are free to Share - copy and redistribute the material in any medium or format - and Adapt the content - remix, transform, and build upon the material for any purpose, even commercially under the following terms:

Attribution: You must give appropriate credit, provide a link to the license, and indicate if changes were made. You may do so in any reasonable manner, but not in any way that suggests the licensor endorses you or your use. 